\title{
An automated method to enrich consumer health vocabularies using GloVe word embeddings and an auxiliary lexical resource
}

\author{
Mohammed Ibrahim ${ }^{\text {Corresp., } 1}{ }^{1}$, Susan Gauch ${ }^{1}$, Omar Salman $^{1}$, Mohammed Alqahtani ${ }^{1}$ \\ ${ }^{1}$ Computer Science and Computer Engineering, University of Arkansas at Fayetteville, Fayetteville, AR, United States \\ Corresponding Author: Mohammed Ibrahim \\ Email address: mohammad.alaubeedy@gmail.com
}

Background: Clear language makes communication easier between any two parties. A layman may have difficulty communicating with a professional due to not understanding the specialized terms common to the domain. In healthcare, it is rare to find a layman knowledgeable in medical terminology which can lead to poor understanding of their condition and/or treatment. To bridge this gap, several professional vocabularies and ontologies have been created to map laymen medical terms to professional medical terms and vice versa.

Objective: Many of the presented vocabularies are built manually or semi-automatically requiring large investments of time and human effort and consequently the slow growth of these vocabularies. In this paper, we present an automatic method to enrich laymen's vocabularies that has the benefit of being able to be applied to vocabularies in any domain.

Methods: Our entirely automatic approach uses machine learning, specifically Global Vectors for Word Embeddings (GloVe), on a corpus collected from a social media healthcare platform to extend and enhance consumer health vocabularies. Our approach further improves the consumer health vocabularies by incorporating synonyms and hyponyms from the WordNet ontology. The basic GloVe and our novel algorithms incorporating WordNet were evaluated using two laymen datasets from the National Library of Medicine (NLM), Open-Access Consumer Health Vocabulary (OAC CHV) and MedlinePlus Healthcare Vocabulary .

Results: The results show that GloVe was able to find new laymen terms with an F-score of $48.44 \%$. Furthermore, our enhanced GloVe approach outperformed basic GloVe with an average F-score of 61\%, a relative improvement of $25 \%$. Furthermore, the enhanced GloVe showed a statistical significance over the two ground truth datasets with $P<.001$.

Conclusions: This paper presents an automatic approach to enrich consumer health vocabularies using the GloVe word embeddings and an auxiliary lexical source, WordNet. Our approach was evaluated used healthcare text downloaded from MedHelp.org, a healthcare social media platform using two standard laymen vocabularies, OAC CHV, and MedlinePlus. We used the WordNet ontology to expand the healthcare corpus by including synonyms, hyponyms, and hypernyms for each layman term occurrence in the corpus. Given a seed term selected from a concept in the ontology, we measured our algorithms' ability to automatically extract synonyms for those terms that appeared in the ground truth concept. We found that enhanced GloVe outperformed GloVe with a relative improvement of $25 \%$ in the F-score. 
2 An Automated Method to Enrich Consumer Health

3 Vocabularies Using GloVe Word Embeddings and An

4 Auxiliary Lexical Resource

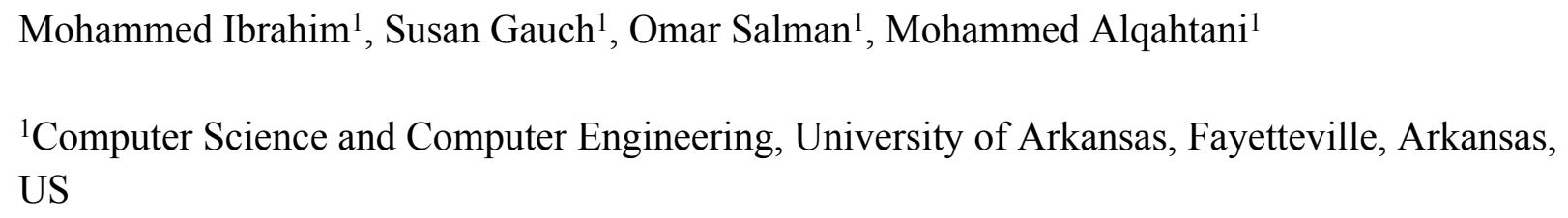

Corresponding Author:

12 Mohammed Ibrahim

131 University of Arkansas, Fayetteville, Arkansas, 72701, United States

14 Email address: mohammad.alaubeedy@gmail.com

\section{Abstract}

Background: Clear language makes communication easier between any two parties. A layman may have difficulty communicating with a professional due to not understanding the specialized terms common to the domain. In healthcare, it is rare to find a layman knowledgeable in medical terminology which can lead to poor understanding of their condition and/or treatment. To bridge this gap, several professional vocabularies and ontologies have been created to map laymen medical terms to professional medical terms and vice versa.

Objective: Many of the presented vocabularies are built manually or semi-automatically requiring large investments of time and human effort and consequently the slow growth of these vocabularies. In this paper, we present an automatic method to enrich laymen's vocabularies that has the benefit of being able to be applied to vocabularies in any domain.

Methods: Our entirely automatic approach uses machine learning, specifically Global Vectors for Word Embeddings (GloVe), on a corpus collected from a social media healthcare platform to extend and enhance consumer health vocabularies. Our approach further improves the consumer health vocabularies by incorporating synonyms and hyponyms from the WordNet ontology. The basic GloVe and our novel algorithms incorporating WordNet were evaluated using two laymen datasets from the National Library of Medicine (NLM), Open-Access Consumer Health Vocabulary (OAC CHV) and MedlinePlus Healthcare Vocabulary.

Results: The results show that GloVe was able to find new laymen terms with an F-score of $48.44 \%$. Furthermore, our enhanced GloVe approach outperformed basic GloVe with an average F-score of $61 \%$, a relative improvement of $25 \%$. Furthermore, the enhanced GloVe showed a statistical significance over the two ground truth datasets with $P<.001$.

Conclusions: This paper presents an automatic approach to enrich consumer health vocabularies using the GloVe word embeddings and an auxiliary lexical source, WordNet. Our approach was evaluated used healthcare text downloaded from MedHelp.org, a healthcare social media platform using two standard laymen vocabularies, OAC CHV, and MedlinePlus. We used the WordNet ontology to expand the healthcare corpus by including 
42 synonyms, hyponyms, and hypernyms for each layman term occurrence in the corpus.

43 Given a seed term selected from a concept in the ontology, we measured our algorithms'

44 ability to automatically extract synonyms for those terms that appeared in the ground truth

45 concept. We found that enhanced GloVe outperformed GloVe with a relative improvement

46 of $25 \%$ in the F-score.

47 Introduction

48 An ontology is a formal description and representation of concepts with their definitions, 49 relations, and classifications in a specific or general domain of discourse [1]. It can decrease

50 terminological and conceptual confusion between system software components and facilitate

51 interoperability. Examples of ontologies in different domains are the BabelNet [2], Arabic

52 Ontology [3], WordNet [4], and Gene Ontology [5]. Ontologies have been used in many domains

53 such as document indexing [6,7], personalizing user's profiles for information retrieval systems

54 [8-13], and providing readable data for semantic web applications [14-17].

55

Several ontologies have been developed and/or proposed for the healthcare domain. One of the biggest healthcare ontologies in the field of biomedicine is the Unified Medical Language system (UMLS). This ontology consists of more than 3,800,000 professional biomedicine concepts. It lists biomedical concepts from different resources, including their part of speech and variant forms [18]. The National Library of Medicine (NLM) manages the UMLS ontology and updates it yearly. As examples of the professional vocabularies included in the UMLS, the Gene Ontology (GO) [5], Disease Ontology (DO) [19], and Medical Subject Headings (MeSH) [7]. The UMLS not only has professional vocabularies but also included laymen vocabularies. These vocabularies provide straightforward terms mapped to the professional medical concepts.

With the advancement of medical technology and the emergence of internet social media, people are more connected than before. In terms of medical technology, there are many efforts to build smart devices that can interact and provide health information. On social media, people started not only sharing their climate concerns, politics, or social problems, but also their health problems. The Pew Research Center conducted a telephone survey in 2010 and reported that $80 \%$ of the United States internet users looked for a healthcare information. The survey showed that $66 \%$ of those users looked for a specific disease or medical issue and roughly $55 \%$ of them looked for a remedies treat to their medical problems [20]. Another study showed that the rate of using social media by physicians grew from $41 \%$ in 2010 to $90 \%$ in 2011 [21-23]. In all these cases, any retrieval system will not be able to interact effectively with laypeople unless they have a lexical source or ontology that defines the medical terminology.

Medical professionals are well-versed in specialized medical terminology developed to be a precise way for healthcare professionals to communicate with each other. However, this medical jargon is obscure to laymen and may require patients to ask for more details to be sure that they understand their condition and treatment plans [24]. A recent study [25] showed the effect of health literacy on the accuracy of the information laymen are seeking related to coronavirus (COVID-19) and cancer. The study concluded that much of the cancer and COVID-19 information available does match with patients' health literacy because much of the present information has been written using professional terminology which is hard for laymen to understand. Having a way to map professional medical terminology to easier to understand 
87 laymen terms could close the communication gap between patients and the healthcare 88 professional.

89

90

91

92

93

94

95

96

97

98

99

100

101

102

103

104

105

106

107

108

109

110

111

112

113

114

115

116

117

118

119

120

121

122

123

124

125

126

127

128

129

130

131

132

Recently, steps have been taken to close the gap between the vocabulary the professionals use in healthcare and what laymen use. It was reported by [26] that approximately five million doctor letters are sent to patients each month. Using words like liver instead of hepatic and brain instead of cerebral could make the doctor's letters much easier to laymen [27]. Thus, the Academy of Medical Royal Colleges started an initiative in 2017 in which the doctors asked to write to patients directly using plain English instead of medical terminology [28]. The twentieth century witnessed steps of building a lot of vocabularies that maps professional medical concepts to their laymen terms and vice versa. These vocabularies are commonly known as consumer health vocabularies. Many of these presented vocabularies are built manually or semiautomatically requiring large investments of time and human effort and consequently these vocabularies grow very slowly. In this paper, we present an automatic method to enrich laymen's vocabularies that has the benefit of being able to be applied to vocabularies in any domain.

\section{Consumer Health Vocabularies}

Consumer health vocabularies can decrease the gap between laymen and professional language and help humans and machines to understand both languages. Zeng et al. reported poor search results when a layman searched for the term heart attack because physicians discuss that condition using the professional concept myocardial infarction [29]. There are many laymen vocabularies proposed in the field of biomedicine such as the Open-Access and Collaborative Consumer Health Vocabulary (OAC CHV) [30], MedlinePlus topics [31], and Personal Health Terminology (PHT) [6]. Those laymen vocabularies should grow from time to time to cover new terms proposed by the laypeople to keep system using them updated.

Our research studies two common English laymen vocabularies, the Open-Access and Collaborative Consumer Health Vocabulary (OAC CHV) and the MedlinePlus vocabularies. The National Library of Medicine (NLM) integrated these vocabularies to the UMLS ontology. Roughly 56,000 UMLS concepts were mapped to OAC CHV concepts. Many of these medical concepts have more than one associated layman term. This vocabulary has been updated many times to include new terms, and the last update was in 2011 [30]. All of the updates incorporated human evaluation of the laymen terms before adding them to the concept. We did an experiment using all 56,000 UMLS concepts that had associated OAC CHV laymen terms. We stemmed, downcased, and removed all stopwords, punctuations, and numbers from the concepts and their laymen terms. We then compared the tokens in the concept names with the set of laymen terms. From this experiment, we found that $48 \%$ of the included laymen terms were just morphological variations of the professional medical terms with minor changes such as replacing lowercase/uppercase letters, switching between plural/single word forms, or adding numbers or punctuation. Table 1 shows a few examples of the laymen and their associated professional UMLS concepts, demonstrating close relationships between the two. The CUI in this table refers to the Concept Unique Identifier that the UMLS uses to identify its biomedicine concepts.

The MedlinePlus vocabulary was constructed to be the source of index terms for the MedlinePlus search engine [31]. The NLM updates this resource yearly. In the 2018 UMLS version, there were 2112 professional concepts mapped to their laymen terms from the MedlinePlus topics. 
133 Due to the extensive human effort required, when we compared the 2018 UMLS version to the 1342020 UMLS version, we found that only 28 new concepts had been mapped to their associated 135 laymen terms. This slow rate of growth motivates the development of tools and algorithms to 136 boost progress in mapping between professional and laymen.

Our research enriches laymen's vocabularies automatically based on healthcare text and seed terms from existing laymen vocabularies. Our system uses the Global Vector for Word Representations (GloVe) to build word embeddings to identify words similar to already existing laymen terms in a consumer health vocabulary. These potential matches are ranked by similarity and top matches are added to their associated medical concept. To improve the identification of new laymen terms, the GloVe results were enhanced by adding hyponyms, hypernyms, and synonyms from a well-known English ontology, WordNet. We make three main contributions:

- Developing an entirely automatic algorithm to enrich consumer health vocabularies automatically by identifying new laymen terms to be added entirely automatically.

- Developing an entirely automatic algorithm to add laymen terms to formal medical concepts that currently have no associated laymen terms.

- Improving GloVe algorithm results by using WordNet with a small, domain-specific corpora to build more accurate word embedding vectors.

152

153

154

Our work differs from others in that it is not restricted to a specific healthcare domain such as Cancer, Diabetes, or Dermatology. Moreover, our improvement is tied to enhancing the text and works with the unmodified GloVe algorithm. This allows for different word embedding algorithms to be applied. Furthermore, expanding small-size corpus with words from standard sources such as the WordNet eliminates the need to download large-size corpus, especially in a domain that is hard to find large text related to it.

\section{Related Work}

158

159

160

161

162

163

164

165

166

167

168

169

170

171

172

173

174

175

176

177

\section{Ontology Creation}

The past few years have witnessed an increased demand for ontologies in different domains [32]. According to (Gruber, 1995), any ontology should comply with criteria such as clarity, coherence, and extensibility to be considered as a source of knowledge that can provide shared conceptualization [33]. However, building ontologies from scratch is immensely time-consuming and requires a lot of human effort [14]. Algorithms that can build an ontology automatically or semi-automatically can help reducing time and labor required to construct that ontology. Zavitsanos et al. presented an automatic method to build an ontology from scratch using text documents. They build that ontology using the Latent Dirichlet Allocation model and an existing ontology [34]. Kietz et al. [35] prototyped a company ontology semi-automatically with the help of general domain ontology and a domain-specific dictionary. They started with a general domain ontology called the GermanNet ontology. There are many other recent works presented to build ontologies from scratch, automatically or semi-automatically such as [36-39].

Ontologies should not be static. Rather, they should grow as their domains develop enriching existing ontologies with new terms and concepts. Agirre et al. (2000) used internet documents to enrich the concepts of WordNet ontology. They built their corpus by submitting the concept's senses along with their information to get the most relevant webpages. They used statistical approaches to rank the new terms [40]. A group at the University of Arkansas applied two approaches to enrich ontologies; 1) a lexical expansion approach using WordNet; and 2) a text 
178 mining approach. They projected concepts and their instances extracted from already existing 179 ontology to the WordNet and selected the most similar sense using distance metrics [41-47]. 180 Recently, Ali and his team employed multilingual ontologies and documents to enrich not only 181 domain-specific ontologies but also multilingual and multi-domain ontologies [48].

\section{Medical Ontologies}

183 The emphasis on developing an Electronic Health Record (EHR) for patients in the United States

184 encouraged the development of medical ontologies to ensure interoperability between multiple

185 medical information systems [49,50]. There are several healthcare vocabularies that provide

186 human and machine-readable medical terminologies. The Systematized Nomenclature of

187 Medicine Clinical Terms, SNOMED CT, is a comprehensive clinical ontology. It contains more

188 than 300,000 professional medical concepts in multiple languages that has been adopted by many

189 healthcare practitioners [50]. Another professional vocabulary is the Royal Society of

190 Chemistry's Name reaction Ontology (RXNO). The RXNO has over 500 reactions describing

191 different chemical reactions that require organic compounds [51]. Recently, He and his team

192 presented the coronavirus ontology with the purpose of providing machine-readable terms

193 related to the coronavirus pandemic that occurs in 2020. This ontology includes all related

194 coronavirus topics such as diagnosis, treatment, transmission, and prevention areas [52].

195 Medical ontologies, like all other ontologies, need to grow and adapt from time to time. Zheng

196 and Wang [53] prototyped the Gene Ontology Enrichment Analysis Software Tool (GOEST). It

197 is a web-based tool that uses a list of genes from the Gene Ontology and enriches them using

198 statistical methods. Recently, Shanavas et al. [54] presented a method to enrich the UMLS

199 concepts with related documents from a pool of professional healthcare documents. Their aim

200 was to provide retrieval systems with more information about medical concepts.

201 Consumer Health Vocabularies

202 Ontologies developed to organize professional vocabularies are of limited benefit in retrieval

203

204

205

206

207

208

209

210

211

212

213

214

215

216

217

218

219

220

221

222 systems used by laypeople. Laymen usually use the lay language to express their healthcare concerns. Having a consumer health vocabulary can bridge the gap between the users' expression of their health questions and documents written using professional language. Consumer Health Vocabularies are particularly difficult to construct because they typically require knowledge of a specialized domain (medicine), a source of laymen's discussion about medicine, and finally a mechanism to map between the two. Zeng et al. detected and mapped a list of consumer-friendly display (CFD) names into their matched UMLS concepts. Their semiautomatic approach used a corpus collected from queries submitted to a MedlinePlus website. Their manual evaluation ended with mapping CFD names to about 1,000 concepts $[55,56]$. Zeng's team continued working on that list of names to build what is called now the OAC CHV. In their last official update to this vocabulary, they were able to define associated laymen terms to about 56,000 UMLS medical concepts [30].

Several methods have been proposed to enrich such consumer vocabulary, such as He et al. [57] who used a similarity-based technique to find a list of similar terms to a seed term collected from the OAC CHV. Gu [58] also tried to enriched the laymen vocabularies leveraging recent word embedding methods. A most recent work presented a method to enhance the consumer health vocabulary by associating laymen terms with relations from the MeSH ontology [59]. Other recent work showed the variety of consumer vocabularies people use when writing their reviews [60] Previous research on enriching consumer health vocabularies were either semi-automatic or

Peer] Comput. Sci. reviewing PDF | (CS-2021:05:60810:1:1:NEW 8 Jul 2021) 
223 it did produce an automatic system accurate enough to be used in practice. Our automatic 224 approach uses a recent word embedding algorithm, GloVe, which is further enhanced by 225 incorporating a lexical ontology, WordNet. We work with gold standard datasets that are already 226 listed on the biggest biomedicine ontology, the UMLS. This paper extends work we published in 227 [61] by including additional datasets for evaluation and incorporating new approaches to 228 improve the GloVe algorithm. These approaches leverage standard auxiliary resources to enrich 229 the occurrence of laymen terms in the corpus. Although there are many large text corpora for 230 general Natural Language Processing (NLP) research, there are far fewer resources specific to 231 the healthcare domain. In order to extract information about domain-specific word usage by laymen for healthcare, we need to construct a domain-specific corpus of laymen's text. To our knowledge, we are the first to leverage MedlinePlus in order to automatically develop consumer health vocabularies.

Finding Synonyms to Enrich Laymen Vocabularies

236 Our work focuses on finding new synonyms, words with the same meaning, to already existing 237 laymen terms. Recent methods of finding synonyms are based on the idea that a word can be 238 defined by its surroundings. Thus, words that appear in similar contexts are likely to be similar in meaning. To study words in text, they need to be represented in a way that allows for computational processing. Word vector representations are a popular technique that represents each word using a vector of feature weights learned from training texts. In general, there are two main vector-learning models. The first models incorporate global matrix factorization whereas the second models focus on local context windows. The global matrix factorization models generally begin by building a corpus-wide co-occurrence matrix and then they apply dimensionality reduction. An early example of this type of model is Latent Semantic Analysis (LSA) [62] and Latent Dirichlet Allocation (LDA) [63]. The context-window models are based on the idea that a word can be defined by its surroundings. An example of such models is the skip-gram model [64] proposed by Mikolov in 2013 and the model proposed by Gauch et al. in [65]. Word2Vec [66], FastText [67], GloVe [68] and WOVe[69] are all examples of vector learning methods that have been shown to be superior to traditional NLP methods in different text mining applications $[55,64]$. Some of these techniques have been applied in the medical field to build medical ontologies, such as [71-75].

Our work focuses on the word similarity task, or specifically word synonyms task. In order to find these synonyms, we leveraged the GloVe algorithm. This algorithm has outperformed many vector learning techniques in the task of finding word similarity [68]. It combines the advantages of two vector learning techniques: global matrix factorization methods and local context window methods [68]. This algorithm has many applications in different fields such as text similarity [76], node representations [77], emotion detection [78] and many others. This algorithm found its way in many biomedicine such as finding semantic similarity [79], extracting Adverse Drug Reactions (ADR) [80], and analyzing protein sequences [81].

GloVe is generally used with very large corpora, e.g., a 2010 Wikipedia corpus ( 1 billion tokens), a 2014 Wikipedia corpus (1.6 billion tokens), and Gigaword 5 (4.3 billion tokens) [68]. In comparison, our corpus is specialized and much smaller, approximately 1,365,000 tokens. To compensate for the relative lack of training text, we incorporate an auxiliary source of vocabulary, WordNet. The WordNet is a machine-readable English ontology proposed by Professor George A. at the Princeton University. The most recent version has about 118,000 
269 synsets (synonyms) of different word categories such as noun, verb, adjective, and adverb. For 270 every synset, WordNet provides a short definition and sometimes an example sentence. It also 271 includes a network of relations between its synsets. The synonyms, antonyms, hyponyms,

272 hypernyms, meronymy's and some others are all semantic relations that WordNet provides [4].

273 WordNet has been used in many fields to help enrich ontologies in different domains such as

274 [82-84].

\section{Methodology}

276 Figure 1 illustrates the main steps of our algorithm. Our method starts with a corpus collected

277 from a healthcare social media platform to be used as the source of the new laymen terms. Using 278 this corpus, the GloVe algorithm builds word embeddings. For every UMLS medical concept, 279 there is a list of its associated laymen terms from which we select a seed terms for the concept.

280 Using the GloVe vectors and a similarity metric, we identify the most similar words to these seed 281 term and choose the top-ranked candidate as a new layman term. The next sections explain the 282 methodology steps in detail.

\section{Healthcare Corpus}

284 To find new laymen terms, we need text documents that can be used as a source of new laymen 285 terms. Because of the specialized nature of medical terminology, we need domain-specific text related to the field of healthcare. MedHelp.org is a healthcare social media platform that provides a question/answer for people who share their healthcare issues. In this platform, the lay language is used more than formal medical terminology. Instead of writing a short query on the internet that may not retrieve what a user is looking for, whole sentences and paragraphs can be posted on such media [85] and other members of the community can provide answers. People might use sentences such as "I can't fall asleep all night" to refer to the medical term "insomnia" and "head spinning a little" to refer to "dizziness" [86]. Such social media can be an excellent source from which to extract new laymen terms.

\section{Seed term list}

295 Our task is to enrich formal medical concepts that already have associated laymen terms by identifying additional related layperson terms. These associated terms are used as seed terms that the system uses to find synonyms and then these synonyms are added to that medical concept. To do so, we need an existing ontology of medical concepts with associated laymen vocabulary. For our experiment, we used two sources of laymen terms: OAC CHV [30], and the MedlinePlus consumer vocabulary [31]. The OAC CHV covers about 56,000 concepts of the UMLS, and the MedlinePlus mapped to about 2,000 UMLS concepts.

\section{Synonym Identification Algorithms}

This paper reports on the results of applying several algorithms to automatically identify synonyms of the seed terms to add to existing laymen's medical concepts. The algorithms we evaluated are described in the next section.

Global Vectors for Word Representations (GloVe)

307 Our baseline approach uses an unmodified version of GloVe to find the new laymen terms 308 trained only on our unmodified corpus. As reported in [68], GloVe starts collecting word 309 contexts using its global word to word co-occurrence matrix. This matrix is a very large and very 310 sparse matrix that is built during a onetime pass over the whole corpus. Given a word to process, 311 i.e., the pivot word, GloVe counts co-occurrences of words around the pivot word within a 
312 window of a given size. As the windows shift over the corpus, the pivot words and contexts 313 around them continually shift until the matrix is complete. GloVe builds word vectors for each 314 word that summarize the contexts in which that word was found. Because the co-occurrence matrix is very sparse. GloVe uses the log bilinear regression model to build reduce the dimensionality of the co-occurrence matrix. This model also optimizes word vectors by tuning its weights and reducing errors iteratively until finding the best word representations. By comparing the seed terms words vectors with all other word vectors using the cosine similarity measure, highly similar words, i.e., potential new laymen terms, can be located. The unmodified GloVe algorithm is our baseline to compare with the GloVe improvement methods.

\section{GloVe with WordNet}

Word embedding algorithms usually use a very large corpus to build their word representations, e.g., 6B words of Google News corpus are used to train the word2vec vectors [66,87]. In the case of a narrow domain such as healthcare, it is hard to find or build an immense corpus, increasing the sparsity of the co-occurrence matrix and impacting the accuracy of the resulting word vectors. Thus, one of our goals is to investigate the ability of an external ontology to increase the accuracy of word embeddings for smaller corpora. In particular, we present methods to exploit a standard English ontology, WordNet, to enhance GloVe's accuracy on a healthcare domain corpus. WordNet provides a network of relations between its relational synsets such as, synonyms, antonyms, hyponyms, hypernyms, meronymy's and some other relations.

In our research, we investigate using the synonym, hyponym, and hypernym relations to augment our corpus prior to running GloVe. We only expand the seed terms in the corpus with their relational synsets. For each seed term, we located the relational synset of interest, e.g., hyponyms we sort them by similarity to the seed term using the Resnik [88] similarity measurement. Then, we limit the list to not exceed the $10^{\text {th }}$ most similar synsets. We split them evenly into two subsets of roughly equal total similarity using a round-robin algorithm. We then expand the corpus by adding the first subset of relational synset words to the corpus prior to each seed term occurrence and the second subset after each seed term occurrence. One of issues that arises when using WordNet is the polysemy of its synsets. Many words are ambiguous and thus map to synsets with different meanings adding noise to the expanded context vectors. By limiting the number of words used from WordNet and incorporating words from the context around the seed term, the effect of noise on GloVe's model is decreased. In future, we could explore using the context words around the seed terms to identify the best synsets to use for expansion. Figure 2 shows the methodology of our system with the WordNet ontology.

Expressing the WordNet method, let $S=\left\{s_{1}, s_{2}, s_{3}, \ldots, s_{n}\right\}$ be a set of $n$ seed terms. Let $T=" w_{1} w_{2}$ $w_{3} \ldots w_{k}$ " be a text of words in the training corpus. Let $X=\left\{x_{1}, x_{2}, x, \ldots, x_{z}\right\}$ be a set of relational synset terms for the seed term $s_{i}$, where $i=0,1,2, \ldots, n$ These relational synsets are sorted according to their degree of similarity to $s_{i}$ using the Resnik similarity measurement [88]. $X$ is sorted according to Resnik score and divided into two sets $X_{1}$ and $X_{2}$. Each set goes to one side of $s_{i}$. Now, let $s_{i}=w_{j+2}$ in T, where $j=0,1,2,3, \ldots, k$. Then, the new text $\hat{T}$ after adding the relational synsets will look like this:

$$
\hat{T}=" \mathrm{w}_{\mathrm{j}} \mathrm{w}_{\mathrm{j}+1} X_{1} \mathrm{w}_{\mathrm{j}+2} X_{2} \ldots \mathrm{w}_{\mathrm{j}+\mathrm{k}} "
$$


355 Further, consider the effect of T-hat on the GloVe cooccurrence vectors. Assume that $\mathrm{s}_{i}$ has the

356

357

358

359

360

361

362

363

364

365

366

367

368

369

370

371

372

373

374

375

376

377

378

379

380

381

382

383

384

385

386

387

388

389

390

391

392

393

394

395

vector $\overrightarrow{V_{s_{i}}}$. Assume that $X$ has the vector $\overrightarrow{V_{X}}$. After expanding the training corpus with the relational synsets, the new vector $\widetilde{V_{s_{i}}}$ will equal:

$$
\widetilde{V_{s_{i}}}=\overrightarrow{V_{s_{i}}}+\overrightarrow{V_{X}}
$$

The co-occurrence weights of relational synsets that are already in the corpus will be increased incrementally in the vector, while those that are new to the corpus will expand the vector and their co-occurrence weight will be calculated according to the co-occurrence with the seed term. The following sections outline the WordNet approach above with the three types of relational synsets we used: synonyms, hyponyms, and hypernyms.

\section{GloVe WordNet Synonyms (GloVeSyno)}

Synonyms are any words that share the same meaning. For example, the words auto, machine, and automobile are all synonyms of the word car. Having synonyms around a seed term adds more information about that seed term and help building more accurate seed term vectors. When a seed term found in the training corpus, WordNet provides a list of its synonyms. These synonyms are sorted according to their degree of similarity to the seed term. After that, the synonyms are divided into two lists and each list go to one side of the seed term. Here is an example that demonstrate this process. Let $T=$ "I had a headache" be a text in the training corpus. $T$ has the seed term $s=$ headache. The WordNet synonyms of this seed term are \{concern, worry, vexation, cephalalgia\}. Sorting this set according to their degree of similarity results the following set: \{worry, cephalalgia, concern, vexation\}. This set is divided in to two sets $\{$ worry, cephalalgia $\}$ and $\{$ concern, vexation $\}$ and added to the left and right of the $s$ in $T$. So, the $\hat{T}$ equals:

$$
\hat{T}=\text { "I had a worry cephalalgia headache concern vexation" }
$$

Assume that the vector of the seed term $s, \overrightarrow{V_{s}}$, before expanding the training corpus looks like this:

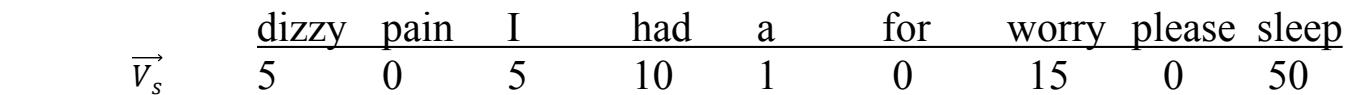

The $\ddot{V_{s}}$ for the seed term after expanding the training corpus with the WordNet synonyms will be expanded to have the new words and updated the occurrence of the already in corpus words. Here is how the $\breve{V_{s}}$ looks like:

$\check{\check{V_{s}}} \begin{array}{cccccccccccc}\text { Cephalalgia } & \text { dizzy } & \text { pain } & \mathrm{I} & \mathrm{had} & \text { a } & \text { for } & \text { concern } & \text { worry } & \text { please } & \text { vexation } & \text { sleep } \\ 1 & 5 & 0 & 5 & 10 & 1 & 0 & 1 & 16 & 0 & 1 & 50\end{array}$

We can see from the $\check{V_{S}}$ that the words that are new to the corpus vocabulary expanded the vector and their weights are calculated according to their co-occurrence with the seed term, while the words that are already in the vector, such as worry, their weights increased incrementally. 
396

397

398

399

400

401

402

403

404

405

406

407

408

409

410

411

412

413

414

415

416

417

418

419

420

421

422

423

424

425

426

427

428

429

430

431

432

433

434

435

436

437

438

439

\section{Glove WordNet Hyponyms (GloVeHypo)}

Hyponyms are those words with more specific meaning, e.g., Jeep is a hyponym of car. The idea here is to find more specific names of a seed term and add them to the context of that seed term. to explain this method, we use the same example we used in the previous section. The hyponyms of the seed term headache that the WordNet provides are \{dead_weight, burden, fardel, imposition, bugaboo, pill, business $\}$. Sorting these hypos according to their degree of similarity to the seed term results the set \{dead_weight, burden, fardel, bugaboo, imposition, business, pill $\}$. This list is divided into two sets and each set go to one of the seed term's sides. The rest process is the same as the GloVeSyno method.

\section{GloVe WordNet Hypernyms (GloVeHyper)}

Hypernyms are the antonyms of hyponyms. Hypernyms are those words with more general meaning, e.g., car is a hypernym of Jeep. The idea here is to surround a seed term with more general information that represents its ontology. Having this information leads to more descriptive vector that represent that seed term. An example of a seed term hypernyms is the hypernyms of the seed term headache, which are \{entity, stimulation, negative_stimulus, information, cognition, psychological feature, abstraction $\}$. We can see that these hypernyms are broader than the seed term headache. We use the same steps for this relational synset as in the GloVeSyno method by sorting, dividing, and distributing these hypernyms around the seed term in the corpus. After that GloVe builds its co-occurrence matrix from the expanded corpus and builds its word vectors that are used to extract the terms most similar terms to the seed terms from the corpus.

\section{Similarity Measurement}

We use cosine similarity between word vectors to find the terms most similar word to the seed term. This metric is widely used for vector comparisons in textual tasks such as [83-86]. Because it focuses on the angle between vectors rather than distances between endpoints, cosine similarity can handle vector divergence in large-size text documents better than Euclidean distance [93]. As such, it is one of the most commonly used similarity based metrics [88-90]. Cosine similarity (Equation 2) produces a score between 0 and 1, and the higher the score between two vectors, the more similar they are[97].

$$
\cos \_\operatorname{sim}\left(v_{1}, v_{2}\right)=\frac{\overrightarrow{v_{1} \cdot \overrightarrow{v_{2}}}}{\left|\overrightarrow{v_{1}}\right|\left|\overrightarrow{v_{2}}\right|},
$$

where $\overrightarrow{v_{1}}$ is a vector of a seed term in the seed term list, and $\overrightarrow{v_{2}}$ is a vector of a word in the corpus that GloVe model built. We consider the terms in the list candidate terms for inclusion. The top $n$ candidate terms are the new laymen terms that we add to the UMLS concept.

\section{Evaluation}

Corpus: MedHelp.org has many communities that discuss different healthcare issues such as Diabetes Mellitus, Heart Diseases, Ear and Eye care, and many others. To select the communities to include in our dataset, we did an informal experiment to find the occurrences of laymen terms from the OAC CHV vocabulary on MedHelp.org. We found that the highest density of these OAC CHV terms occur in communities such as Pregnancy, Women's Health, Neurology, Addiction, Hepatitis-C, Heart Disease, Gastroenterology, Dermatology, and Sexually Transmitted Diseases and Infections (STDs / STIs) communities. We thus chose these nine communities for our testbed and downloaded all the user-posted questions and their answers

PeerJ Comput. Sci. reviewing PDF | (CS-2021:05:60810:1:1:NEW 8 Jul 2021) 
440 from MedHelp.org from WHEN to April 20, 2019. The resulting corpus is roughly $1.3 \mathrm{~Gb}$ and 441 contains approximately 135,000,000 tokens. Table 2 shows the downloaded communities with 442 their statistics.

443

444

We removed all stopwords, numbers, and punctuations from this corpus. We also removed corpus-specific stopwords such as test, doctor, symptom, and physician. Within our domainspecific corpus, these ubiquitous words have little information content. Finally, we stemmed the text using the Snowball stemmer [98], and removed any word less than 3 characters long. The final corpus size was $\sim 900 \mathrm{mb}$. This corpus is available for download from [99].

457

Seed terms: We built the seed term list from the OAC CHV and MedlinePlus vocabularies, choosing seed terms with a unigram form, such as flu, fever, fatigue, and swelling. There are two reason that led us to choose only unigrams for our work. First, GloVe embeddings handle only single word vectors, and second the existing laymen vocabularies are rich with such seed terms. We also chose the professional medical concepts that had a unigram form, then we pick its unigram associated laymen terms. In many cases, the medical concept on these two vocabularies has associate laymen terms that have same names as the concept's name except different morphological forms, such the plural ' $\mathrm{s}$ ', uppercase/lowercase of letters, punctuations, or numbers. We treated these cases and removed any common medical words. After that, we stemmed the terms and listed only the unique terms. For example, the medical concept Tiredness has the laymen terms fatigue, fatigues, fatigued and fatiguing. After stemming, only the term 'fatigu' was kept. To focus on terms for which sufficient contextual data was available, we kept only these laymen terms that occur in the corpus more than 100 times.

To validate our system, we need at least two terms for each professional medical concept, one term is used as the seed laymen term and one to be used as the target term for evaluation. Thus, we kept only those medical concepts that have at least two related terms. From the two vocabularies, we were able to create an OAC CHV ground truth dataset of size 944 medical concepts with 2103 seed terms and a MedlinePlus ground truth dataset of size 101 medical concepts with 227 seed terms. Table 3 shows an example of some UMLS medical concepts and their seed terms from the MedlinePlus dataset. When we run our experiments, we select one seed term at random from each of the 944 concepts in the ground truth dataset for testing. We evaluate each algorithm's ability to identify that seed term's ground truth synonyms using the metrics described in the next section.

The OAC CHV dataset is nine times bigger than the MedlinePlus dataset (see Figure 3a); the OAC CHV vocabulary covers 56,000 of the UMLS concepts whereas the MedlinePlus covers only 2,112 UMLS concepts. Although it is smaller, MedlinePlus represents the future of laymen terms because the NLM updates this resource annually. In contrast, the last update to the OAC $\mathrm{CHV}$ was in 2011. Figure $3 \mathrm{~b}$ shows that $37 \%$ of the 101 concepts in MedlinePlus also appear in the OAC CHV dataset and share the same concepts and laymen terms. This indicates that the $\mathrm{OAC} \mathrm{CHV}$ is still a good source of laymen terms.

Baselines and metrics. We consider the basic GloVe results as the baseline for comparison with the WordNet expansion algorithms. First, we tune the baseline to the best setting. We then compare the results when we use those settings with our WordNet-expanded corpora. We 
486 evaluate our approach using precision $(\mathrm{P})$, recall $(\mathrm{R})$, and F-score $(\mathrm{F})$, which is the harmonic

487

488

489

490

491

492

493

494

495

496

497

498

499

500

501

502

503

504

505

506

507

508

509

510

511

512

513

514

515

516

517

518

519

520

521

522

523

524 mean of the previous two [100]. We also include the number of concepts (NumCon) that the system could find one or more of its seed terms. Moreover, we include the Mean Reciprocal Rank (MRR) [101] that measures the rank of the first most similar candidate term in the candidate list. It has a value between 0 and 1 , and the closer the MRR to 1 , the closer the candidate term position in the candidate list.

Based on a set of medical concepts for which we have a seed term and at least one synonym in the ground truth data set, we can measure the precision, recall, and F-Score metrics according to two criteria: (1) the number of concepts for which the system was able to find at least one synonym; and (2) the total number of synonyms for seed terms the system was able to find across all concepts. We call the metrics used to measure these two criteria the macro and micro average metrics, respectively. The macro average measures the number of the concepts for which the algorithm found a match to the ground truth dataset while the micro average measures the number of new terms found. The micro and macro precision, recall, and F-score are computed according to these equations:

$$
P_{\text {micro }}=\frac{\# \text { of true synonyms in the candidate lists }}{\text { total } \# \text { of terms in the candidate lists }},
$$

\# of true synonyms in the candidate lists

$$
R_{\text {micro }}=\frac{\text { \# of true synonyms in the candidate lists }}{\text { total \# of synonms in the ground truth dataset }},
$$

$$
P_{\text {macro }}=\frac{\# \text { of concepts whose candidate list contains a true synonym }}{\text { total \# of concepts }},
$$

We illustrate these measurements in the following example. Suppose we have a ground truth dataset of size 25 concepts, and every concept has four synonyms terms. For every concept, a random synonym term selected to be a seed term. The remaining 75 synonyms will be used for evaluation. Suppose the algorithm retrieves five candidate terms for each seed term and it is able to generate results for 20 of the seed terms, creating 20 candidate term lists. That makes 100 candidate terms in total. Assume that only 15 out of the 20 candidate lists contain a true synonym, and each list of those 15 lists includes two true synonyms. Thus, this algorithm extracted 30 true laymen terms. Having all this information, then the $P_{\text {micro }}=30 / 100, R_{\text {micro }}=$ $30 / 75, P_{\text {macro }}=15 / 20$, and $R_{\text {macro }}=15 / 25$.

\section{Results}

\section{Experiment 1: Tuning GloVe to the Best Setting}

To tune the GloVe algorithm to its best setting, we varied GloVe's parameters on an unmodified corpus and we used the larger of our two datasets, the OAC CHV for testing. The GloVe algorithm has many hyperparameters, but the vector size and the window size parameters have the biggest effect on the results. We evaluated GloVe using the 944 concepts in this dataset on different vector sizes $(100,200,300,400)$, varying the window size $(10,20,30,40)$ for each 
525

vector size. We set the candidate list size to $n=10$. Figure 4 shows the macro F-score results of the GloVe algorithm according to these different vector and window sizes. In general, the Fscore results declined with any window size greater than 30 .

Table 4 reports the micro-precision for GloVe over the same parameter settings. We can see that the micro precision is very low due to the size of the candidate lists created. In particular, we are testing with 944 concept seed terms, and the size of the candidate list is set to 10 , so we generate $944 \times 10=9440$ candidate terms. However, there are only 2103 truth synonyms, to the microaverages are guaranteed to be quite low. To compensate, we need to determine a good size for the candidate list that balances recall and precision. This is discussed further in Section 5.4.

The highest F-score was reported with a vector of size 400 and window of size 30 . Thus, we used these settings for all following experiments.

\section{Experiment 2: GloVe with WordNet}

Using the best GloVe setting reported in the previous experiment, we next evaluate the GloVeSyno, GloVeHypo, and GloVeHyper algorithms to determine whether or not they can improve on basic GloVe's ability to find layman terms. After processing the corpus, we expand our laymen's corpus with synonyms, hyponyms, and hypernyms from WordNet, respectively. These are then input to the GloVe algorithm using 400 for the vector size and 30 for the window size. Table 5 shows a comparison between the results of these WordNet algorithms, and our baseline GloVe for the OAC CHV and MedlinePlus datasets. The evaluation was done using a candidate list of size $n=10$. We report here the macro accuracy of the system for the three algorithms which is based on the number of concepts for which a ground truth result was found.

We can see from Table 5 that GloVeSyno outperformed the other algorithms. It was able to enrich synonyms to $57 \%$ (546) of the medical concepts listed in the OAC CHV dataset and more than $62 \%$ (63) of the concepts in the MedlinePlus dataset. Table 6 presents the algorithms' performance averaged over the two datasets. On average, the GloVeSyno algorithm produced an F-score relative improvement of $25 \%$ comparing to the basic GloVe. Moreover, the GloVeSyno reported the highest MRR over all the other algorithms, which shows that the first most similar candidate term to the seed term fell approximately in the $2^{\text {nd }}$ position of the candidate list. Furthermore, the GloVeSyno showed a high statistical significance over the two ground truth datasets with $P<.001$.

The GloVeHypo and GloVeHyper results were not good comparing to the other algorithms. The reason is that the hyponyms provide a very specific layman term synsets. For example, the hyponyms of the laymen term edema are angioedema, atrophedema, giant hives, periodic edema, Quincke', papilledema, and anasarca. Such hypos are specific names of the laymen term edema, and they might not be listed in ground truth datasets. We believe that the GloVeHypo algorithm results are promising, but a more generalized and bigger size ground truth dataset is required to prove that.

On the other hand, the GloVeHyper algorithm was not good comparing to the basic GloVe algorithm. However, it is better than the GloVeHypo algorithm. The reason that this algorithm did not get a good result is because the degree of abstraction that the hypernym relations provide. For example, the hypernym contagious_disease represents many laymen terms, such as $f l u$, 
571 rubeola, and scarlatina. Having such hypernym in the context of a layman term did not lead to 572 good results. The hypernym contagious_disease is very general relation that can represent 573 different kind of diseases.

574

575

576

577

578

579

580

581

582

583

584

585

586

587

588

589

590

591

592

593

594

595

596

597

598

599

600

601

602

603

604

605

606

607

608

609

610

611

612

613

614

615

616

To illustrate the effectiveness of the GloVeSyno algorithm, we show a seed term the candidate synonyms for a selection of concepts in Table 7 . The candidate synonyms that appear in the ground truth list of synonyms are shown bolded. Although only 14 true synonyms from 7 concepts were found, we note that many of the other candidate synonyms seem to be good matches even though they do not appear in the official vocabulary. These results are promising and could be used to enrich medical concepts with missing laymen terms. They could also be used by healthcare retrieval systems to direct laypersons to the correct healthcare topic.

On average, the GloVeSyno algorithm outperformed all the others, producing an F-score relative improvement of $25 \%$ compared to basic GloVe. The results were statistically significant $(\mathrm{p}<.001)$. Additionally, this algorithm found many potentially relevant laymen terms that were not already in the ground truth.

We further examined the effect of the WordNet terms on the set of candidate terms extracted from the corpus. With GloVeSyno, $60 \%$ of the true positives appeared in the WordNet synsets used for corpus expansion versus $41 \%$ with unmodified GloVe. Thus, even after expanding with WordNet, $40 \%$ of the true positives appeared in the corpus and not in the nearest WordNet synsets, indicating that the external lexicon and GloVe's word vectors find complementary sets of synonyms.

\section{Experiment 3: Improving the GloVeSyno Micro Accuracy}

From our previous experiment, we conclude that the GloVeSyno algorithm was the most effective. However, we next explore it in more detail to see if we can improve its accuracy by selecting an appropriate number of candidate synonyms from the candidate lists. We report evaluation results according to the ground truth datasets, OAC CHV and MedlinePlus. We varied the number of synonyms selected from the candidate lists $n=1$ to $n=100$ and measured the micro recall, precision, and F-score. Figure 5 shows the F-score results and the number of concepts for which at least one true synonym was extracted. This figure reports the results of the GloVeSyno algorithm over the OAC CHV dataset. The F-score is maximized with $n=3$ with an F-score of $19.06 \%$ and 365 out of 944 concepts enriched. After that, it starts to decline quickly and at $n=20$ the F-score is only $6.75 \%$ which further declines to $1.7 \%$ at $n=100$. We note that the number of concepts affected rose quickly until $n=7$, but then grows more slowly. The best results are with $n=2$ with an F-score of $19.11 \%$. At this setting, 287 of the 944 concepts are enriched with a micro-precision of $15.43 \%$ and recall of $25.11 \%$.

The evaluation results over the MedlinePlus dataset looks the same as the results reported for the OAC CHV dataset (See Figure 6). The F-score was at its highest score at $n=2$ with an F-score of $23.12 \%$ and 33 out of 101 concepts enriched. The F-score decreased quickly at $n=30$ and was at its lowest score at $n=100$ with an F-score of $1.81 \%$. The number of enriched concepts grew quickly until $n=6$ and stabilized after $n=9$ between 64 and 74 enriched concepts.

Over the two datasets, the best results are with $n=2$. Figure 7 shows the F-score over the Precision and recall for the two datasets. Despite the difference in the number of concepts

Peer] Comput. Sci. reviewing PDF | (CS-2021:05:60810:1:1:NEW 8 Jul 2021) 
617 between the two ground truth datasets, the results show that the F-score is the best at $n=2$. The

618 figure shows that the behaviors of the GloVeSyno over the two datasets are almost the same over 619 different candidate list settings.

620

621

622

623

Based on these results, we conclude that the best performance for automatically enriching a laymen vocabulary with terms suggested by GloVeSyno be achieved by adding the top two results.

624

625

626

627

628

629

630

631

632

633

634

635

636

637

638

639

640

641

642

643

644

645

646

647

648

649

650

651

652

653

654

655

656

657

658

659

\section{Conclusion and Future Work}

This paper presents an automatic approach to enrich consumer health vocabularies using GloVe word embeddings and an auxiliary lexical source, WordNet. Our approach was evaluated used a healthcare text downloaded from MedHelp.org, a healthcare social media platform using two standard laymen vocabularies, OAC CHV, and MedlinePlus. We used the WordNet ontology to expand the healthcare corpus by including synonyms, hyponyms, and hypernyms for each layman term occurrence in the corpus. Given a seed term selected from a concept in the ontology, we measured our algorithms' ability to automatically extract synonyms for those terms that appeared in the ground truth concept. We found that GloVeSyno and GloVeHypo both outperformed GloVe on the unmodified corpus, however including hypernyms actually degraded performance. GloVeSyno was the best performing algorithm with a relative improvement of $25 \%$ in the F-score versus the basic GloVe algorithm. Furthermore, the GloVeSyno showed a high statistical significance over the two ground truth datasets with $\mathrm{P}<.001$.

The results of the system were in general promising and can be applied not only to enrich laymen vocabularies for medicine but any ontology for a domain, given an appropriate corpus for the domain. Our approach is applicable to narrow domains that may not have the huge training corpora typically used with word embedding approaches. In essence, by incorporating an external source of linguistic information, WordNet, and expanding the training corpus, we are getting more out of our training corpus.

For the future work, we plan to use our expanded corpus to train and evaluate the state of the art word embedding algorithms, such as BERT [102], GPT-2 [103], CTRL [104], and GPT-3 [105]. Furthermore, we plan to use our collected ground truth datasets to evaluate the recent work done by Huang and his team that is called ClinicalBERT [106]. Also, for the future work, we plan to do further improvements to the GloVeSyno, GloVeHypo, GloVeHyper algorithms and test them using the UMLS semantics to explore more laymen terms relationships. In our experiments, we implemented our algorithms on only unigram seed terms. We plan to explore applying these algorithms to different word grams of different lengths. Moreover, in our work, we used the MedHelp.org corpus to find new laymen term. Even though this corpus was rich with laymen information, our plan is to use larger healthcare dataset and might apply to multilanguage datasets to find laymen terms in different languages. In addition, we are currently exploring an iterative feedback approach to expand the corpus with words found by GloVe itself rather than those in an external linguistic resource. We are also working on our other project that tackle the problem of adding these laymen terms that laypeople use but not covered in the laymen vocabularies. 
660 References

661 1. Grüninger M, Fox MS. Methodology for the design and evaluation of ontologies. 1995;

662 2. Navigli R, Ponzetto SP. BabelNet: The automatic construction, evaluation and application of a

663

664

665

666

667

668

669

670

671

672

673

674

675

676

677

678

679

680

681

682

683

684

685

686

687

688

689

690

691

692

693

694

695

696

697

698

699

700

701

702

703

704

705

706

707

3. Jarrar M. Building a Formal Arabic Ontology (Invited Paper). In proceedings of the Experts Meeting on Arabic Ontologies and Semantic Networks. Alecso, Arab League. Tunis: sn; 2011.

4. Miller GA. WordNet: a lexical database for English. Commun ACM. 1995;38(11):39-41.

5. Consortium GO. The gene ontology project in 2008. Nucleic Acids Res.

2007;36(suppl_1):D440-4.

6. product description: personal health terminology (PHT). Intelligent Medical Objects, Inc.;

7. Lipscomb CE. Medical subject headings (MeSH). Bull Med Libr Assoc. 2000;88(3):265.

8. Gauch S, Chaffee J, Pretschner A. Ontology-based personalized search and browsing. :17.

9. Pretschner A, Gauch S. Ontology based personalized search. In: Proceedings 11th International Conference on Tools with Artificial Intelligence [Internet]. Chicago, IL, USA: IEEE Comput. Soc; 1999 [cited 2019 Sep 26]. p. 391-8. Available from:

http://ieeexplore.ieee.org/document/809829/

10. Trajkova J, Gauch S. Improving ontology-based user profiles. In: Coupling approaches, coupling media and coupling languages for information retrieval. LE CENTRE DE HAUTES ETUDES INTERNATIONALES D'INFORMATIQUE DOCUMENTAIRE; 2004. p. 380-90.

11. Chaffee J, Gauch S. Personal ontologies for web navigation. In: Proceedings of the ninth international conference on Information and knowledge management. ACM; 2000. p. 227-34.

12. Challam V, Gauch S, Chandramouli A. Contextual search using ontology-based user profiles. In: Large Scale Semantic Access to Content (Text, Image, Video, and Sound). LE CENTRE DE HAUTES ETUDES INTERNATIONALES D'INFORMATIQUE DOCUMENTAIRE; 2007. p. 612-7.

13. Challam VKR, Gauch S. Contextual information retrieval using ontology based user profiles [PhD Thesis]. University of Kansas, Electrical Engineering and Computer Science; 2004.

14. Maedche A, Staab S. Ontology learning for the semantic web. IEEE Intell Syst. 2001;16(2):729.

15. Fensel D, Van Harmelen F, Horrocks I, McGuinness DL, Patel-Schneider PF. OIL: An ontology infrastructure for the semantic web. IEEE Intell Syst. 2001;16(2):38-45.

16. Doan A, Madhavan J, Domingos P, Halevy A. Learning to map between ontologies on the semantic web. In: Proceedings of the 11th international conference on World Wide Web. AcM; 2002. p. 662-73.

17. McIlraith SA, Son TC, Zeng H. Semantic web services. IEEE Intell Syst. 2001;16(2):46-53.

18. Unified Medical Language System (UMLS) [Internet]. [cited 2019 Oct 21]. Available from: https://www.nlm.nih.gov/research/umls/index.html

19. Schriml LM, Arze C, Nadendla S, Chang Y-WW, Mazaitis M, Felix V, Feng G, Kibbe WA. Disease Ontology: a backbone for disease semantic integration. Nucleic Acids Res. 2011;40(D1):D9406.

20. Fox S. Health Topics [Internet]. Pew Research Center: Internet, Science \& Tech. 2011 [cited 2019 Oct 21]. Available from: https://www.pewinternet.org/2011/02/01/health-topics-3/

21. Bosslet GT, Torke AM, Hickman SE, Terry CL, Helft PR. The patient-doctor relationship and online social networks: results of a national survey. J Gen Intern Med. 2011;26(10):1168-74.

22. George DR, Rovniak LS, Kraschnewski JL. Dangers and opportunities for social media in medicine. Clin Obstet Gynecol. 2013;56(3).

23. Modahl M, Tompsett L, Moorhead T. Doctors, Patients \& Social Media. Soc Media. 2011;16.

24. Josh K. WHAT IS MEDICAL JARGON? | Century Fitness [Internet]. 2017 [cited 2021 Feb 27]. Available from: http://centuryfit.com/what-is-medical-jargon/ 
708

709

710

711

712

713

714

715

716

717

718

719

720

721

722

723

724

725

726

727

728

729

730

731

732

733

734

735

736

737

738

739

740

741

742

743

744

745

746

747

748

749

750

751

752

753

754

755

756

25. Papadakos J, Reznikov V, Giannopoulos E, Giuliani M, Papadakos T. The Literacy Demand of Cancer \& COVID-19 Consumer Health Information. J Consum Health Internet. 2021 Jan 2;25(1):50-64.

26. Blanchard S. Doctors told to write to their patients in plain English [Internet]. Daily Mail Online. 2018 [cited 2019 Oct 21]. Available from: http://www.dailymail.co.uk/health/article6129323/Doctors-told-write-patients-plain-English.html

27. Doctors told to use "plain English." BBC News [Internet]. 2018 Sep 4 [cited 2019 Oct 21]; Available from: https://www.bbc.com/news/health-45394620

28. Yeginsu C. Rx for British Doctors: Use Plain English Instead of Latin. The New York Times [Internet]. 2018 Sep 5 [cited 2019 Oct 21]; Available from: https://www.nytimes.com/2018/09/05/world/europe/doctors-plain-english.html

29. Zeng Q, Kogan S, Ash N, Greenes RA. Patient and clinician vocabulary: how different are they? Medinfo. 2001;10(Pt 1):399-403.

30. Doing-Harris KM, Zeng-Treitler Q. Computer-assisted update of a consumer health vocabulary through mining of social network data. J Med Internet Res. 2011;13(2):e37.

31. Miller N, Lacroix E-M, Backus JEB. MEDLINEplus: building and maintaining the National Library of Medicine's consumer health Web service. Bull Med Libr Assoc. 2000 Jan;88(1):11-7.

32. Bautista-Zambrana MR. Methodologies to build ontologies for terminological purposes. Procedia-Soc Behav Sci. 2015;173:264-9.

33. Gruber TR. Toward principles for the design of ontologies used for knowledge sharing? Int J Hum-Comput Stud. 1995;43(5-6):907-28.

34. Zavitsanos E, Paliouras G, Vouros GA, Petridis S. Discovering Subsumption Hierarchies of Ontology Concepts from Text Corpora. In: IEEE/WIC/ACM International Conference on Web Intelligence (WI'07). 2007. p. 402-8.

35. Kietz J-U, Maedche A, Volz R. A Method for Semi-Automatic Ontology Acquisition from a Corporate Intranet. 2000 Oct;15.

36. Hier DB, Brint SU. A Neuro-ontology for the neurological examination. BMC Med Inform Decis Mak. 2020 Mar 4;20(1):47.

37. Yilahun H, Imam S, Hamdulla A. Ontology expansion based on UWN reusability. Int J Inf Commun Technol. 2020 Jan 1;16(4):339-52.

38. Sanagavarapu LM, Iyer V, Reddy YR. OntoEnricher: A Deep Learning Approach for Ontology Enrichment from Unstructured Text. ArXiv Prepr ArXiv210204081. 2021;

39. Mellal N, Guerram T, Bouhalassa F. An Approach for Automatic Ontology Enrichment from Texts. Informatica. 2021;45(1).

40. Agirre E, Ansa O, Hovy E, Martinez D. Enriching very large ontologies using the WWW. arXiv:cs/0010026 [Internet]. 2000 Oct 17 [cited 2019 Sep 24]; Available from: http://arxiv.org/abs/cs/0010026

41. Luong HP, Gauch S, Wang Q. Ontology learning through focused crawling and information extraction. In: 2009 International Conference on Knowledge and Systems Engineering. IEEE; 2009. p. 106-12.

42. Luong H, Gauch S, Wang Q. Ontology learning using word net lexical expansion and text mining. Theory Appl Adv Text Min. 2012;101.

43. Wang Q, Gauch S, Luong H. Ontology concept enrichment via text mining. In: IADIS international conference on internet technologies \& society. 2010. p. 147-54.

44. Luong HP, Gauch S, Speretta M. Enriching concept descriptions in an amphibian ontology with vocabulary extracted from wordnet. In: 2009 22nd IEEE International Symposium on Computer-Based Medical Systems. IEEE; 2009. p. 1-6.

45. Luong HP, Gauch S, Wang Q. Ontology-based focused crawling. In: 2009 International Conference on Information, Process, and Knowledge Management. IEEE; 2009. p. 123-8.

Peer] Comput. Sci. reviewing PDF | (CS-2021:05:60810:1:1:NEW 8 Jul 2021) 
757

758

759

760

761

762

763

764

765

766

767

768

769

770

771

772

773

774

775

776

777

778

779

780

781

782

783

784

785

786

787

788

789

790

791

792

793

794

795

796

797

798

799

800

801

802

803

804

805

46. Luong H, Gauch S, Wang Q, Maglia A. An ontology learning framework using focused crawler and text mining. Int J Adv Life Sci. 2009;1(2):99-109.

47. Speretta M, Gauch S. Using text mining to enrich the vocabulary of domain ontologies. In: Proceedings of the 2008 IEEE/WIC/ACM International Conference on Web Intelligence and Intelligent Agent Technology-Volume 01. IEEE Computer Society; 2008. p. 549-52.

48. Ali M, Fathalla S, Ibrahim S, Kholief M, Hassan YF. CLOE: a cross-lingual ontology enrichment using multi-agent architecture. Enterp Inf Syst. 2019 Sep 14;13(7-8):1002-22.

49. Rector AL, Qamar R, Marley T. Binding ontologies and coding systems to electronic health records and messages. Appl Ontol. 2009;4(1):51-69.

50. Donnelly K. SNOMED-CT: The advanced terminology and coding system for eHealth. Stud Health Technol Inform. 2006;121:279.

51. Schneider N, Lowe DM, Sayle RA, Tarselli MA, Landrum GA. Big data from pharmaceutical patents: a computational analysis of medicinal chemists' bread and butter. J Med Chem. 2016;59(9):4385-402.

52. He Y, Yu H, Ong E, Wang Y, Liu Y, Huffman A, Huang H, Beverley J, Hur J, Yang X, Chen L, Omenn GS, Athey B, Smith B. CIDO, a community-based ontology for coronavirus disease knowledge and data integration, sharing, and analysis. Sci Data. 2020 Jun 12;7(1):181.

53. Zheng Q, Wang X-J. GOEAST: a web-based software toolkit for Gene Ontology enrichment analysis. Nucleic Acids Res. 2008 Jul 1;36(suppl_2):W358-63.

54. Shanavas N, Wang H, Lin Z, Hawe G. Ontology-based enriched concept graphs for medical document classification. Inf Sci. 2020 Jul 1;525:172-81.

55. Zeng QT, Tse T, Crowell J, Divita G, Roth L, Browne AC. Identifying Consumer-Friendly Display (CFD) Names for Health Concepts. AMIA Annu Symp Proc. 2005;2005:859-63.

56. Zeng QT, Tse T. Exploring and developing consumer health vocabularies. J Am Med Inform Assoc. 2006;13(1):24-9.

57. He Z, Chen Z, Oh S, Hou J, Bian J. Enriching consumer health vocabulary through mining a social Q\&A site: A similarity-based approach. J Biomed Inform. 2017;69:75-85.

58. Gu G, Zhang X, Zhu X, Jian Z, Chen K, Wen D, Gao L, Zhang S, Wang F, Ma H, Lei J. Development of a Consumer Health Vocabulary by Mining Health Forum Texts Based on Word Embedding: Semiautomatic Approach. JMIR Med Inform. 2019;7(2):e12704.

59. Monselise M, Greenberg J, Liang OS, Pascua S, Kim H, Kelly M, Boone JP, Yang CC. An Automatic Approach to Extending the Consumer Health Vocabulary. J Data Inf Sci. 2021;6(1):35.

60. Hovy D, Melumad S, Inman JJ. Wordify: a Tool for Discovering and Differentiating Consumer Vocabularies. J Consum Res. 2021;

61. Ibrahim M, Gauch S, Salman O, Alqahatani M. Enriching Consumer Health Vocabulary Using Enhanced GloVe Word Embedding. ArXiv200400150 Cs Stat [Internet]. 2020 Apr 13 [cited 2020 May 31]; Available from: http://arxiv.org/abs/2004.00150

62. Deerwester S, Dumais ST, Furnas GW, Landauer TK, Harshman R. Indexing by latent semantic analysis. J Am Soc Inf Sci. 1990;41(6):391-407.

63. Blei DM, Ng AY, Jordan MI. Latent dirichlet allocation. J Mach Learn Res. 2003;3(Jan):9931022.

64. Mikolov T, Yih W, Zweig G. Linguistic regularities in continuous space word representations. In: Proceedings of the 2013 Conference of the North American Chapter of the Association for Computational Linguistics: Human Language Technologies. 2013. p. 746-51.

65. Gauch S, Wang J, Rachakonda SM. A corpus analysis approach for automatic query expansion and its extension to multiple databases. ACM Trans Inf Syst TOIS. 1999;17(3):250-69.

66. Mikolov T, Sutskever I, Chen K, Corrado GS, Dean J. Distributed representations of words and phrases and their compositionality. In: Advances in neural information processing systems. 2013. p. 3111-9.

Peer] Comput. Sci. reviewing PDF | (CS-2021:05:60810:1:1:NEW 8 Jul 2021) 
806

807

808

809

810

811

812

813

814

815

816

817

818

819

820

821

822

823

824

825

826

827

828

829

830

831

832

833

834

835

836

837

838

839

840

841

842

843

844

845

846

847

848

849

850

851

852

853

854

855

67. Bojanowski P, Grave E, Joulin A, Mikolov T. Enriching word vectors with subword information. Trans Assoc Comput Linguist. 2017;5:135-46.

68. Pennington J, Socher R, Manning C. Glove: Global vectors for word representation. In: Proceedings of the 2014 conference on empirical methods in natural language processing (EMNLP). 2014. p. 1532-43.

69. Ibrahim M, Gauch S, Gerth T, Cox B. WOVe: Incorporating Word Order in GloVe Word Embeddings. ArXiv210508597 Cs [Internet]. 2021 May 18 [cited 2021 Jul 1]; Available from: http://arxiv.org/abs/2105.08597

70. Hasan SA, Farri O. Clinical Natural Language Processing with Deep Learning. In: Consoli S, Reforgiato Recupero D, Petković M, editors. Data Science for Healthcare [Internet]. Cham: Springer International Publishing; 2019 [cited 2019 Oct 3]. p. 147-71. Available from: http://link.springer.com/10.1007/978-3-030-05249-2_5

71. Minarro-Giménez JA, Marin-Alonso O, Samwald M. Exploring the application of deep learning techniques on medical text corpora. Stud Health Technol Inform. 2014;205:584-8.

72. Hughes M, Li I, Kotoulas S, Suzumura T. Medical text classification using convolutional neural networks. Stud Health Technol Inf. 2017;235:246-50.

73. De Vine L, Zuccon G, Koopman B, Sitbon L, Bruza P. Medical semantic similarity with a neural language model. In: Proceedings of the 23rd ACM international conference on conference on information and knowledge management. ACM; 2014. p. 1819-22.

74. Minarro-Giménez JA, Marín-Alonso 0, Samwald M. Applying deep learning techniques on medical corpora from the world wide web: a prototypical system and evaluation. ArXiv Prepr ArXiv150203682. 2015;

75. Wang C, Cao L, Zhou B. Medical synonym extraction with concept space models. In: TwentyFourth International Joint Conference on Artificial Intelligence. 2015.

76. Kenter T, De Rijke M. Short text similarity with word embeddings. In: Proceedings of the 24th ACM international on conference on information and knowledge management. 2015. p. 141120.

77. Brochier R, Guille A, Velcin J. Global vectors for node representations. In: The World Wide Web Conference. 2019. p. 2587-93.

78. George A, HB BG, Soman KP. Teamcen at semeval-2018 task 1: global vectors representation in emotion detection. In: Proceedings of the 12th international workshop on semantic evaluation. 2018. p. 334-8.

79. Muneeb TH, Sahu S, Anand A. Evaluating distributed word representations for capturing semantics of biomedical concepts. In: Proceedings of BioNLP 15. 2015. p. 158-63.

80. Lin W-S, Dai H-J, Jonnagaddala J, Chang N-W, Jue TR, Iqbal U, Shao JY-H, Chiang I-J, Li Y-C. Utilizing different word representation methods for twitter data in adverse drug reactions extraction. In: 2015 Conference on Technologies and Applications of Artificial Intelligence (TAAI). IEEE; 2015. p. 260-5.

81. George A, Ganesh HB, Kumar MA, Soman KP. Significance of global vectors representation in protein sequences analysis. In: Computer Aided Intervention and Diagnostics in Clinical and Medical Images. Springer; 2019. p. 261-9.

82. Espinoza M, Gómez-Pérez A, Mena E. Enriching an ontology with multilingual information. In: European Semantic Web Conference. Springer; 2008. p. 333-47.

83. Navigli R, Velardi P. Enriching a formal ontology with a thesaurus: an application in the cultural heritage domain. In: Proceedings of the 2 nd workshop on ontology learning and population: Bridging the gap between text and knowledge. 2006. p. 1-9.

84. Warin M, Oxhammar H, Volk M. Enriching an ontology with wordnet based on similarity measures. 2005;

85. Kilicoglu H, Abacha AB, Mrabet Y, Shooshan SE, Rodriguez L, Masterton K, Demner-Fushman D. Semantic annotation of consumer health questions. BMC Bioinformatics. 2018;19(1):34. 
856

857

858

859

860

861

862

863

864

865

866

867

868

869

870

871

872

873

874

875

876

877

878

879

880

881

882

883

884

885

886

887

888

889

890

891

892

893

894

895

896

897

898

899

900

901

902

903

904

905

86. Tutubalina E, Miftahutdinov Z, Nikolenko S, Malykh V. Medical concept normalization in social media posts with recurrent neural networks. J Biomed Inform. 2018;84:93-102.

87. Mikolov T, Chen K, Corrado G, Dean J. Efficient estimation of word representations in vector space. ArXiv Prepr ArXiv13013781. 2013;

88. Resnik P. Using information content to evaluate semantic similarity in a taxonomy. ArXiv Prepr Cmp-Lg9511007. 1995;

89. Salton G, Wong A, Yang CS. A vector space model for automatic indexing. Commun ACM. 1975 Nov 1;18(11):613-20.

90. Habibi M, Cahyo PW. Journal Classification Based on Abstract Using Cosine Similarity and Support Vector Machine. JISKA J Inform Sunan Kalijaga. 2020;4(3):185-92.

91. Park K, Hong JS, Kim W. A methodology combining cosine similarity with classifier for text classification. Appl Artif Intell. 2020;34(5):396-411.

92. Zheng L, Jia K, Bi T, Fang Y, Yang Z. Cosine Similarity Based Line Protection for Large Scale Wind Farms. IEEE Trans Ind Electron. 2020;

93. Prabhakaran S. Cosine Similarity - Understanding the math and how it works? (with python) [Internet]. ML+. 2018 [cited 2021 Mar 1]. Available from: https://www.machinelearningplus.com/nlp/cosine-similarity/

94. Lüthe M. Calculate Similarity - the most relevant Metrics in a Nutshell [Internet]. Medium. 2019 [cited 2021 Mar 1]. Available from: https://towardsdatascience.com/calculatesimilarity-the-most-relevant-metrics-in-a-nutshell-9a43564f533e

95. Gupta S. Top 5 Distance Similarity Measures implementation in Machine Learning [Internet]. 2019 [cited 2021 Mar 1]. Available from: https://medium.com/@gshriya195/top-5-distancesimilarity-measures-implementation-in-machine-learning-1f68b9ecb0a3

96. Polamuri S. Five most popular similarity measures implementation in python [Internet]. Dataaspirant. 2015 [cited 2021 Mar 1]. Available from: https://dataaspirant.com/five-mostpopular-similarity-measures-implementation-in-python/

97. Singhal A. Modern information retrieval: A brief overview. IEEE Data Eng Bull. 2001;24(4):3543.

98. Porter MF. Snowball: A language for stemming algorithms. 2001.

99. Enrich and Expand Consumer Health Vocabularies Using GloVe Word Embeddings [Internet]. [cited 2021 Mar 27]. Available from: http://text.csce.uark.edu/chv.html

100. Powers DM. Evaluation: from precision, recall and F-measure to ROC, informedness, markedness and correlation. 2011;

101. Voorhees EM, Harman DK. The Eighth Text REtrieval Conference (TREC-8). 2000 Nov 1 [cited 2020 Oct 29]; Available from: https://www.nist.gov/publications/eighth-text-retrievalconference-trec-8

102. Devlin J, Chang M-W, Lee K, Toutanova K. BERT: Pre-training of Deep Bidirectional Transformers for Language Understanding. ArXiv181004805 Cs [Internet]. 2019 May 24 [cited 2020 Mar 18]; Available from: http://arxiv.org/abs/1810.04805

103. Radford A, Wu J, Child R, Luan D, Amodei D, Sutskever I. Language Models are Unsupervised Multitask Learners. 2019;24.

104. Keskar NS, McCann B, Varshney LR, Xiong C, Socher R. CTRL: A Conditional Transformer Language Model for Controllable Generation. ArXiv190905858 Cs [Internet]. 2019 Sep 20 [cited 2021 Feb 8]; Available from: http://arxiv.org/abs/1909.05858

105. Brown TB, Mann B, Ryder N, Subbiah M, Kaplan J, Dhariwal P, Neelakantan A, Shyam P, Sastry G, Askell A, Agarwal S, Herbert-Voss A, Krueger G, Henighan T, Child R, Ramesh A, Ziegler DM, Wu J, Winter C, Hesse C, Chen M, Sigler E, Litwin M, Gray S, Chess B, Clark J, Berner C, McCandlish S, Radford A, Sutskever I, Amodei D. Language Models are Few-Shot Learners. ArXiv200514165 Cs [Internet]. 2020 Jul 22 [cited 2021 Feb 28]; Available from: http://arxiv.org/abs/2005.14165

Peer] Comput. Sci. reviewing PDF | (CS-2021:05:60810:1:1:NEW 8 Jul 2021) 
906 106. Huang K, Altosaar J, Ranganath R. ClinicalBERT: Modeling Clinical Notes and Predicting

907

908 Hospital Readmission. ArXiv190405342 Cs [Internet]. 2020 Nov 28 [cited 2021 Mar 22];

909 Available from: http://arxiv.org/abs/1904.05342 
Figure 1

Methodology of finding new laymen terms.

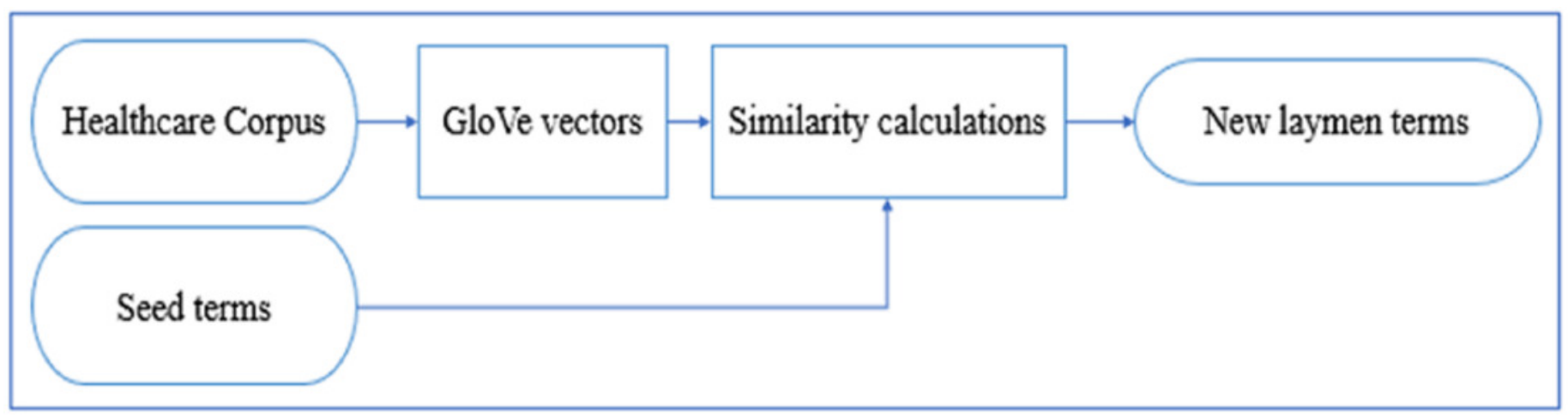


Figure 2

Methodology of improved Glove with WordNet corpus enhancement.

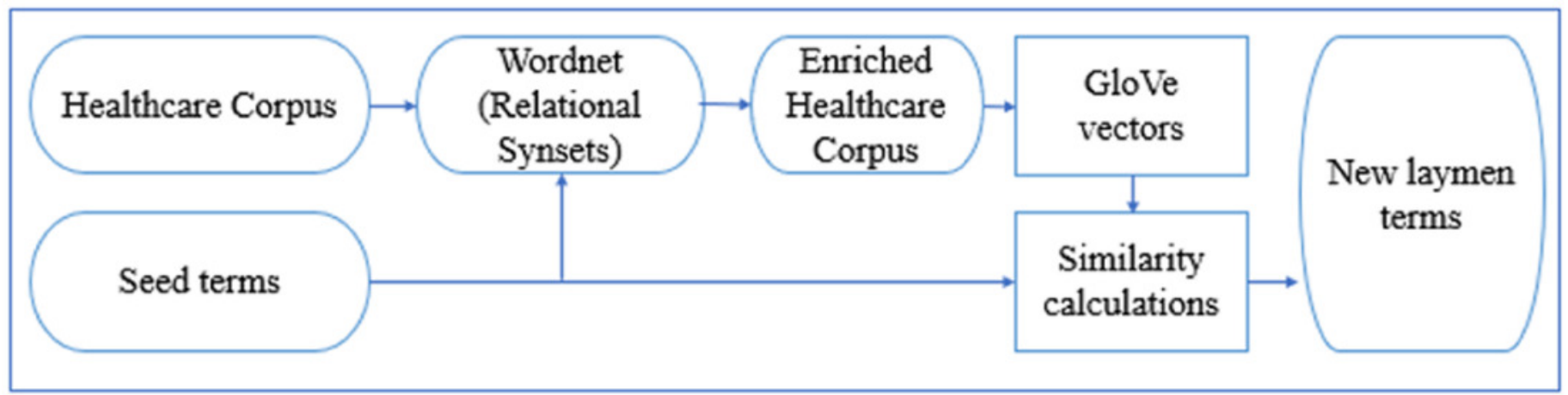


Figure 3

a. Size of the OAC CHV dataset to the MedlinePlus dataset. b. Shared concepts and their laymen terms between the MedlinePlus and OAC CHV datasets.
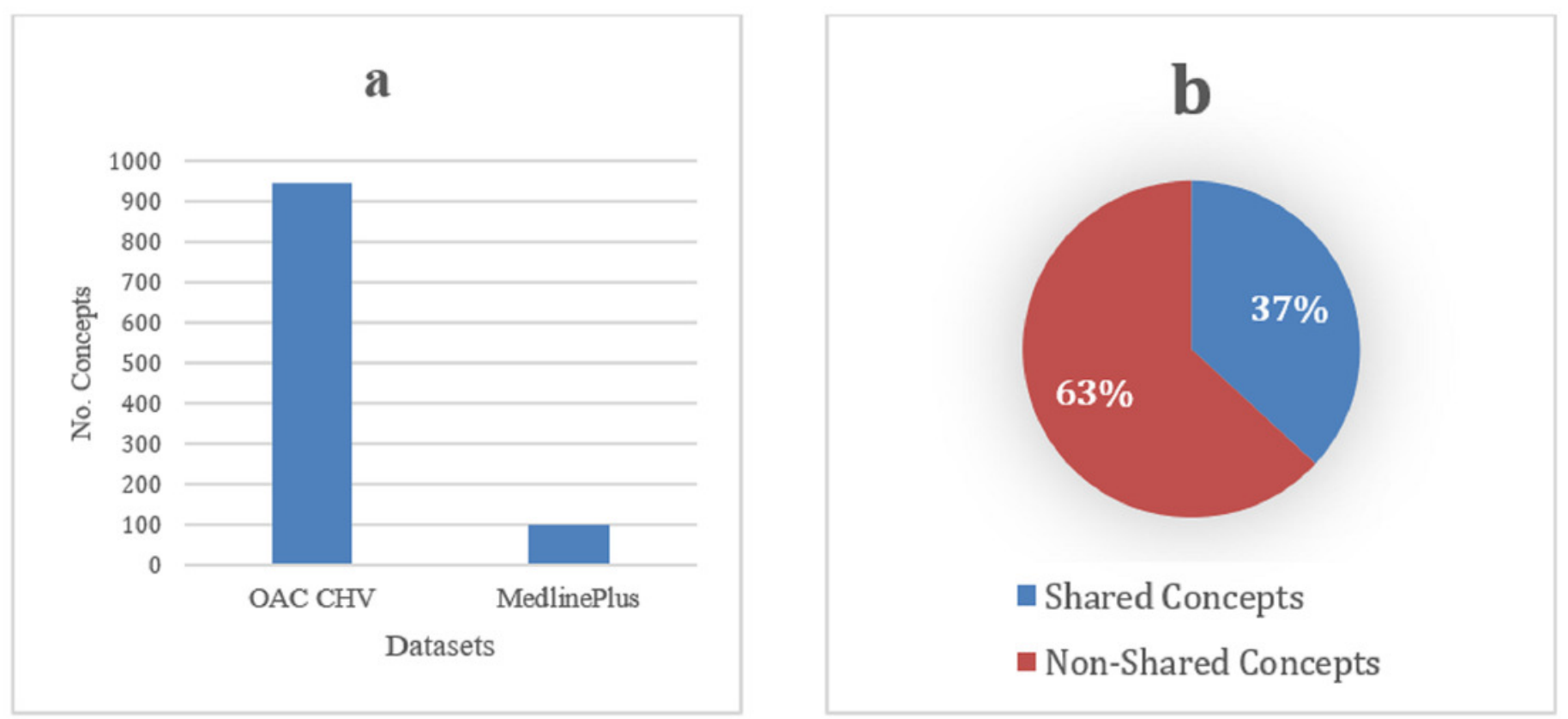
Figure 4

The Macro F-Score for the GloVe algorithm with Different Vector and Window Sizes.

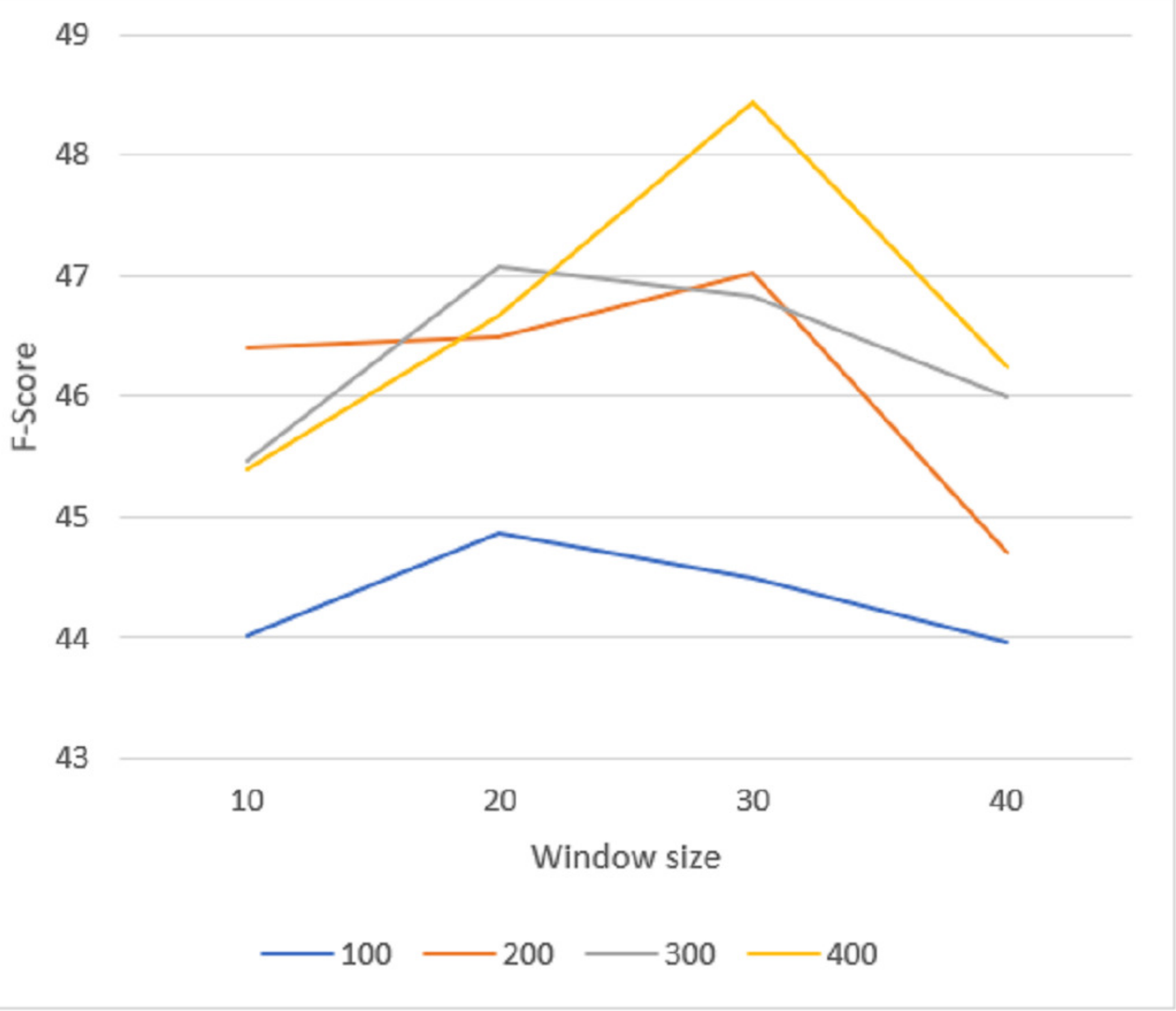


Figure 5

Micro F-Score and the number of concepts for the GloVeSyno algorithm over the OAC CHV dataset.

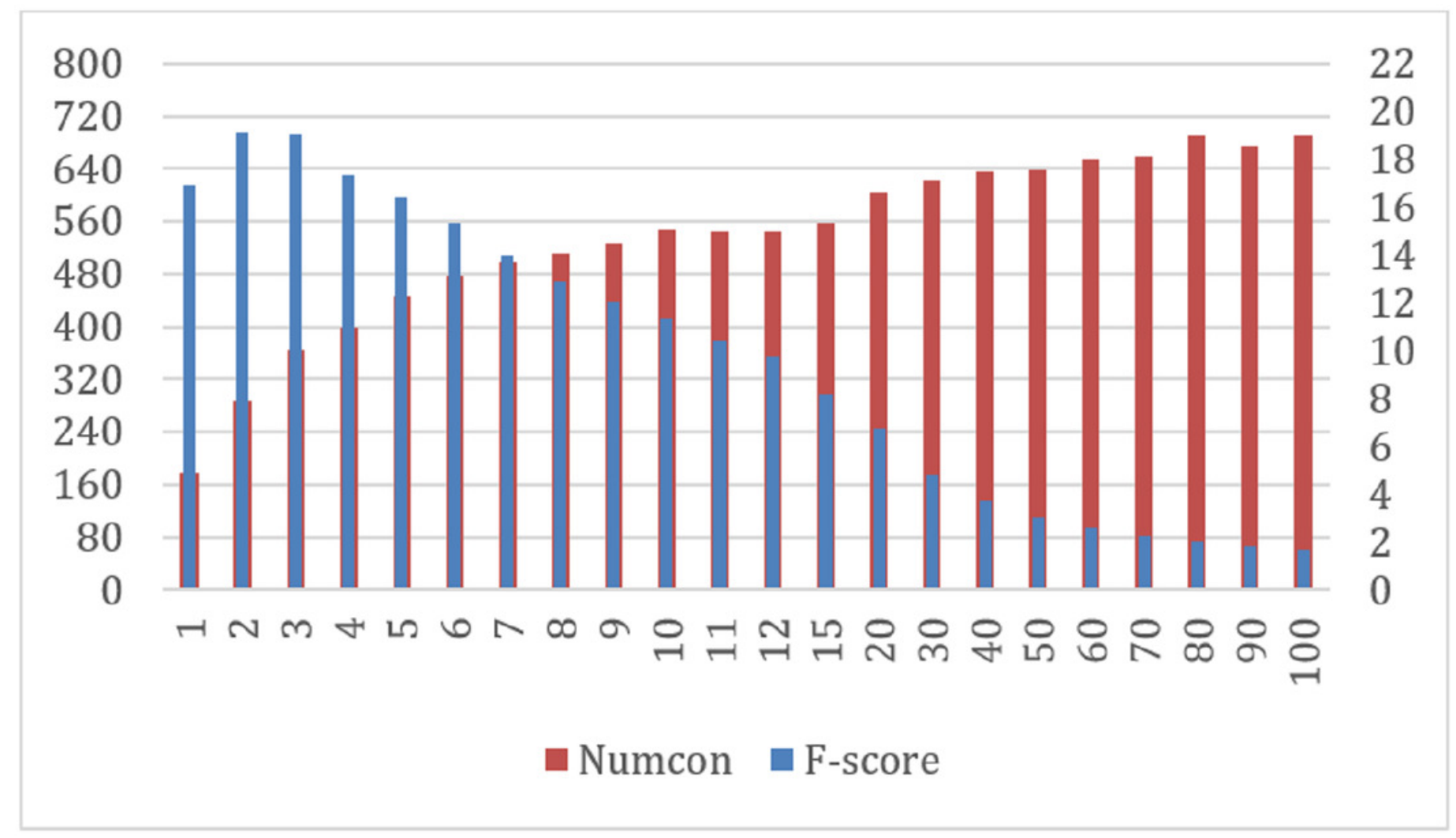


Figure 6

Micro F-Score and the number of concepts for the GloVeSyno algorithm over the MedlinePlus dataset.

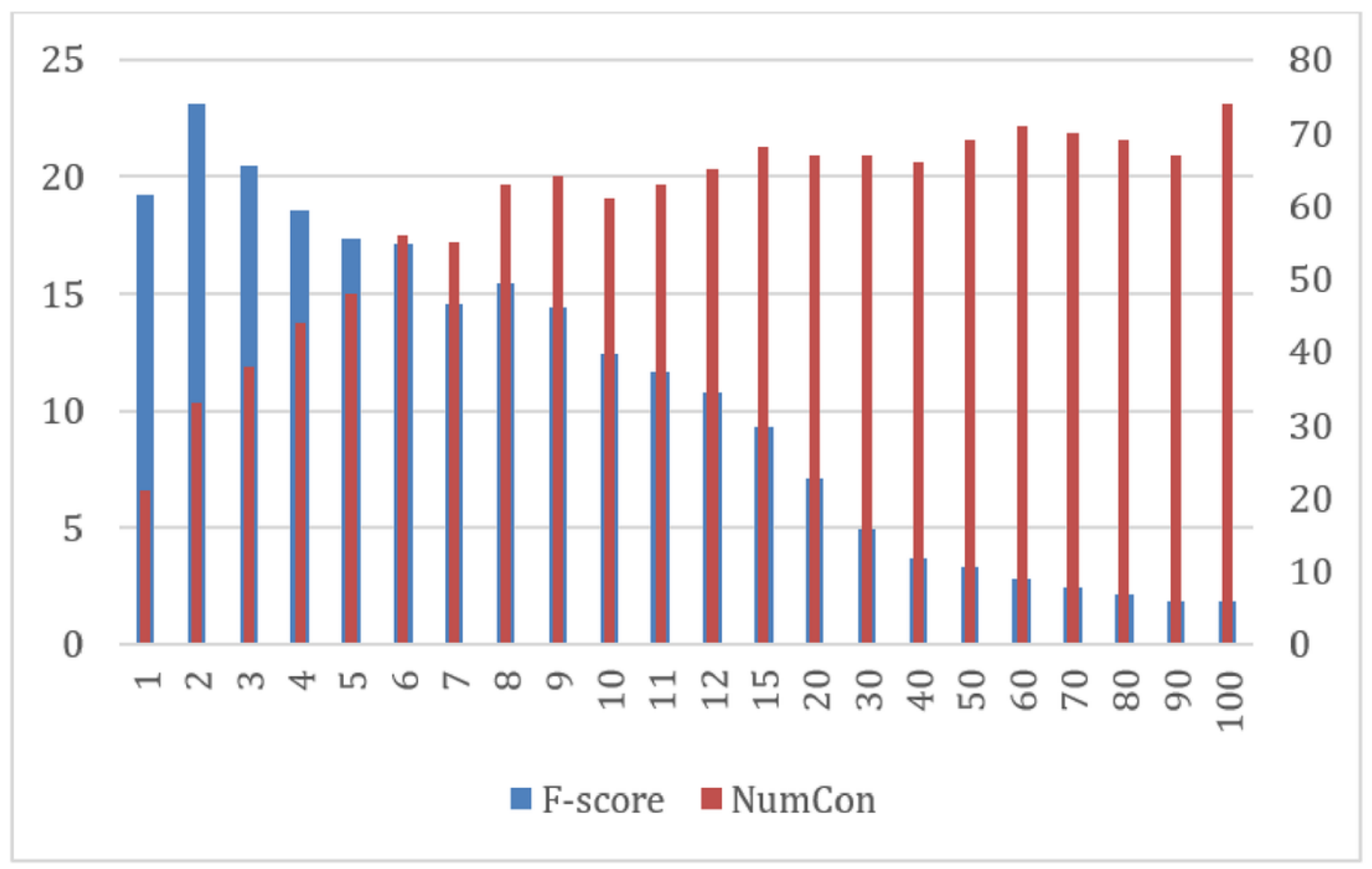


Figure 7

$a$ and $b$ F-Score results over the Precision and Recall for the GloVeSyno algorithm over the OAC CHV and MedlinePlus datasets.

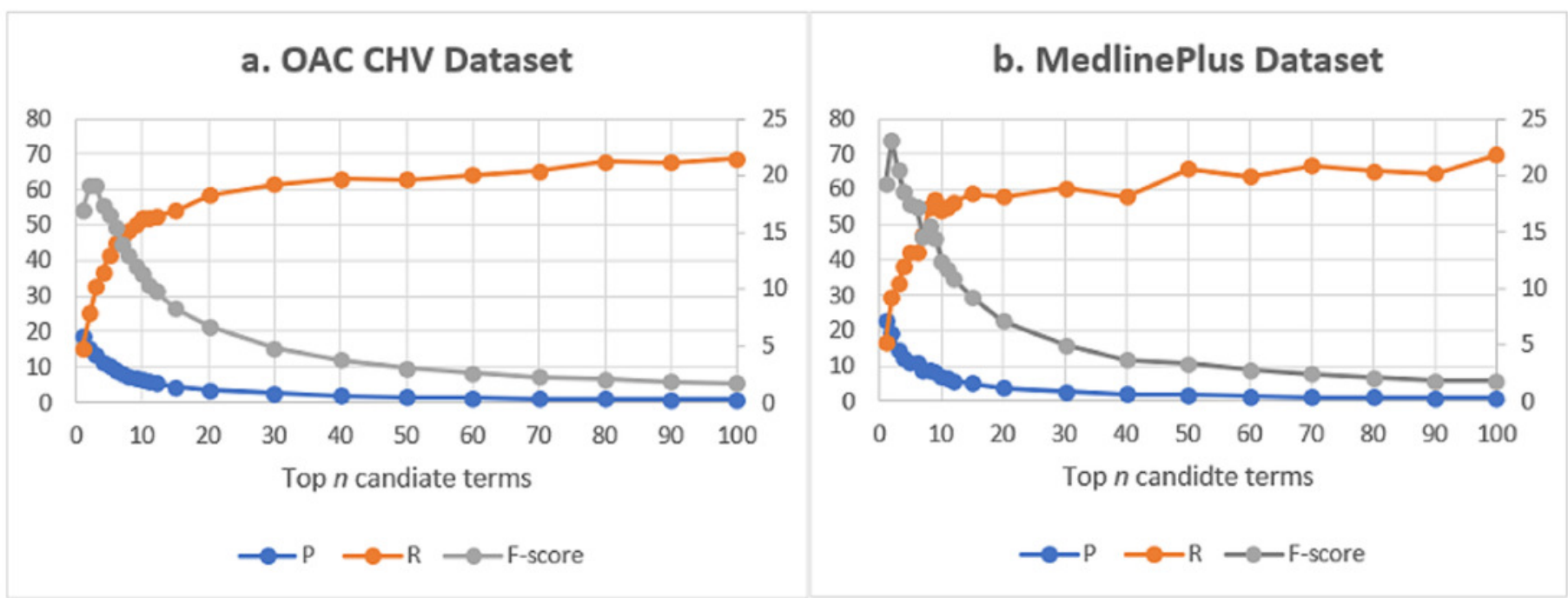




\section{Table 1 (on next page)}

Sample UMLS concepts and some of their OA CHV associated laymen terms. 


\begin{tabular}{|l|l|l|l|l|l|}
\hline CUI & UMLS Concept & \multicolumn{3}{|l|}{ Associated laymen terms } \\
\hline C0018681 & Headache & headache & headaches & head ache & ache head \\
\hline C0003864 & Arthritis & arthritis & arthritides & arthritide & \\
\hline C0033860 & Psoriasis & Psoriasis & psoriasi & psoriasis & \\
\hline
\end{tabular}

1 


\section{Table 2 (on next page)}

MedHelp.org Community Corpus Statistics. 


\begin{tabular}{|r|l|l|l|}
\hline No. & Community & Posts & Tokens \\
\hline 1. & Addiction & 82,488 & $32,871,561$ \\
\hline 2. & Pregnancy & 308,677 & $33,989,647$ \\
\hline 3. & Hepatitis-C & 46,894 & $21,142,999$ \\
\hline 4. & Neurology & 62,061 & $9,394,044$ \\
\hline 5. & Dermatology & 67,109 & $8,615,484$ \\
\hline 6. & STDs / STIs & 59,774 & $7,275,289$ \\
\hline 8. & Gastroenterology & 43,394 & $6,322,356$ \\
\hline 9. & Women health & 66,336 & $5,871,323$ \\
\hline 10. & Heart Disease & 33,442 & $5,735,739$ \\
\hline 11. & Eye Care & 31,283 & $4,281,328$ \\
\hline & Total & 801,458 & $135,499,770$ \\
\hline
\end{tabular}

1 


\section{Table 3 (on next page)}

UMLS concepts with their seed terms from the MedlinePlus dataset. 


\begin{tabular}{|l|l|l|l|l|}
\hline CUI & Medical Concept & \multicolumn{3}{|l|}{ Associated Laymen Terms } \\
\hline C0043246 & laceration & lacer & torn & tear \\
\hline C0015672 & fatigue & weariness & tired & fatigued \\
\hline C0021400 & influenza & flu & influenza & grippe \\
\hline
\end{tabular}




\section{Table 4 (on next page)}

The micro-precision of GloVe. 


\begin{tabular}{|c|c|c|c|c|}
\hline & \multicolumn{3}{|c|}{ Micro } \\
\hline Vector Size & NumCon & P & R & F \\
\hline $\mathbf{1 0 0}$ & 420 & 4.78 & 38.91 & 8.51 \\
\hline $\mathbf{2 0 0}$ & 444 & 5.07 & 41.33 & 9 \\
\hline $\mathbf{3 0 0}$ & 442 & 5.16 & 42.02 & 9.19 \\
\hline $\mathbf{4 0 0}$ & $\mathbf{4 5 7}$ & $\mathbf{5 . 2 8}$ & $\mathbf{4 2 . 9 7}$ & $\mathbf{9 . 4 1}$ \\
\hline
\end{tabular}

1 


\section{Table 5 (on next page)}

Evaluation of the basic GloVe, GloVeSyno, GloVeHypo, and GloVeHyper algorithms over the OAC CHV and MedlinePlus datasets. 


\begin{tabular}{|c|c|c|c|c|c|}
\hline & \multicolumn{5}{|c|}{ Macro } \\
\hline OAC CHV & NumCon & P & R & F & MRR \\
\hline Basic GloVe & 457 & 48.46 & 48.41 & 48.44 & 0.29 \\
\hline GloVeSyno & $\mathbf{5 4 6}$ & $\mathbf{5 7 . 9}$ & $\mathbf{5 7 . 8 4}$ & $\mathbf{5 7 . 8 7}$ & $\mathbf{0 . 3 5}$ \\
\hline GloVeHypo & 280 & 29.69 & 29.66 & 29.68 & 0.33 \\
\hline GloVeHyper & 433 & 45.92 & 45.87 & 45.89 & 0.35 \\
\hline MedlinePlus & \multicolumn{5}{|l}{} \\
\hline Basic GloVe & 48 & 51.06 & 47.52 & 49.23 & 0.38 \\
\hline GloVeSyno & $\mathbf{6 3}$ & $\mathbf{6 6 . 3 2}$ & $\mathbf{6 2 . 3 8}$ & $\mathbf{6 4 . 2 9}$ & $\mathbf{0 . 3 6}$ \\
\hline GloVeHypo & 32 & 33.33 & 31.68 & 32.49 & 0.37 \\
\hline GloVeHyper & 35 & 37.23 & 34.65 & 35.9 & 0.35 \\
\hline
\end{tabular}

1 


\section{Table 6(on next page)}

The average results of the basic GloVe, GloVeSyno, GloVeHypo, and GloVeHyper algorithms over the OAC CHV and MedlinePlus datasets. 


\begin{tabular}{|c|c|c|c|c|c|c|}
\hline & \multicolumn{5}{|c|}{ Macro } & \\
\hline Algorithm & NumCon & P & R & F & MRR & F-score Rel-Improv. \\
\hline Basic GloVe & 252.5 & 49.76 & 47.965 & 48.835 & 0.335 & \\
\hline GloVeSyno & $\mathbf{3 0 4 . 5}$ & $\mathbf{6 2 . 1 1}$ & $\mathbf{6 0 . 1 1}$ & $\mathbf{6 1 . 0 8}$ & $\mathbf{0 . 3 5 5}$ & $\mathbf{2 5 \%}$ \\
\hline GloVeHypo & 156 & 31.51 & 30.67 & 31.085 & 0.350 & $-36 \%$ \\
\hline GloVeHyper & 234 & 41.575 & 40.26 & 40.895 & 0.350 & $-16 \%$ \\
\hline
\end{tabular}

1 


\section{Table 7 (on next page)}

Sample of the GloVeSyno output (seeds stemmed). 


\begin{tabular}{|l|l|l|l|l|l|l|l|l|}
\hline CUI & $\begin{array}{l}\text { Seed } \\
\text { Term }\end{array}$ & \multicolumn{6}{l|}{ Candidate Synonyms } \\
\hline C0015967 & feverish & febric & febril & pyrexia & fever & chili_pepp & chilli & influenza \\
\hline C0020505 & overeat & gormand & pig_out & ingurgit & gormandis & scarf_out & overindulg & gourmand \\
\hline C0013604 & edema & oedema & hydrop & dropsi & swell & puffi & ascit & crestless \\
\hline C0039070 & syncop & swoon & deliquium & faint & vasovag & neurocardi & dizzi & lighthead \\
\hline C0015726 & fear & fright & afraid & scare & terrifi & scari & panic & anxieti \\
\hline C0014544 & seizur & rictus & seiz & raptus & prehend & shanghaier & seizer & clutch \\
\hline C0036916 & stds & std & gonorrhea & encount & chlamydia & hiv & herp & syphili \\
\hline
\end{tabular}

1 